\title{
Hausdorff Dimension of the Range and the Graph of Stable-Like Processes
}

\author{
Xiaochuan Yang ${ }^{1}$ (D)
}

Received: 6 February 2017 / Revised: 29 June 2017

(C) Springer Science+Business Media, LLC 2017

\begin{abstract}
We determine the Hausdorff dimension for the range of a class of pure jump Markov processes in $\mathbb{R}^{d}$, which turns out to be random and depends on the trajectories of these processes. The key argument is carried out through the SDE representation of these processes. The method developed here also allows to compute the Hausdorff dimension for the graph.
\end{abstract}

Keywords Markov processes · Lévy processes · Hausdorff dimension

Mathematics Subject Classification (2010) $60 \mathrm{H} 10 \cdot 60 \mathrm{~J} 25 \cdot 60 \mathrm{~J} 75 \cdot 28 \mathrm{~A} 78$

\section{Introduction}

The range of various stochastic processes provides interesting examples of random fractals. The determination of their Hausdorff dimension is a natural question. For Lévy processes, this question has been addressed by different authors, see for instance Taylor [21], Blumenthal and Getoor [2], McKean [14], Pruitt [17], Khoshnevisan, Xiao and Zhong [9] and the survey article by Xiao [24] for an exhaustive list of literature on this topic. In particular, Pruitt [17] characterized the Hausdorff dimension for the range of general Lévy processes in terms of their potential operators, while Khoshnevisan, Xiao and Zhong [9] measured their range in terms of the characteristic exponent.

This work was supported by grants from Région Ile-de-France.

\footnotetext{
$\bowtie \quad$ Xiaochuan Yang

xiaochuan.j.yang@gmail.com

1 Department of Statistics and Probability, Michigan State University, East Lansing, MI 48824, USA
} 
Recently, there has been much interest in understanding Markov processes generated by pseudo-differential operators; we refer the readers to the monograph by Jacob [7] and a recent survey book by Böttcher, Schilling and Wang [4] (We adopt the terminology "Lévy-type processes" therein). These processes are usually spatially inhomogeneous which is an important feature because real-life data (e.g., financial, geographical and meteorologic data) which have been modeled by Lévy processes often exhibit different characteristics in different locations. Therefore, modeling with Lévy-type processes can be relevant.

The determination of the Hausdorff dimension of Lévy-type processes, contrary to the Lévy case, seems to be far from being accomplished. In particular, only upper (see Schilling [18]) and lower bounds (see Knopova, Schilling and Wang [10]) are known under various conditions and these bounds do not match in general.

The purpose of this article is to find the exact Hausdorff dimension of the sample paths of a specific class of Lévy-type processes in $\mathbb{R}^{d}$, called stable-like processes, whose generator can be written for all $f \in C_{c}^{2}\left(\mathbb{R}^{d}\right)$, twice continuously differentiable functions with compact support,

$$
\mathcal{L}^{\beta} f(x)=\int\left[f(x+u)-f(x)-1_{|u| \leq 1} u \cdot \nabla f(x)\right] \beta(x)|u|^{-d-\beta(x)} \mathrm{d} u,
$$

where $|\cdot|$ is the Euclidean norm in $\mathbb{R}^{d}$ and $\beta$ is a Lipschitz map from $\mathbb{R}^{d}$ to a compact subset of $(0,2)$. This function $\beta$ is the key which gives all the information on dimensional properties of stable-like processes. The uniqueness in law of a Markov process with generator (1) was proved by Bass [1, page 274]. Actually, Bass showed the uniqueness in law for a large class of Lévy-type operators under quite weak regularity conditions on the jump kernel (Dini-continuity for $\beta$ in the stable-like case). Moreover, stable-like processes are Feller processes [1, page 285], so the strong Markov property holds. Here, we assume stronger regularity condition (Lipschitz continuity) because we will use a jump SDE representation of stable-like processes (especially the pathwise uniqueness). We refer the readers to the monograph by Kolokoltsov [12, Chapter 7] for more on distributional properties of stable-like processes, e.g., heat kernel estimates.

The main result of this paper is the following.

Theorem 1 Let $M$ be a stable-like process in $\mathbb{R}^{d}$ that is a Markov process with generator (1). Then a.s. for every open interval $I=(a, b) \subset \mathbb{R}^{+}$,

$$
\operatorname{dim}_{\mathcal{H}}(M(I))=d \wedge \sup _{s \in I} \beta\left(M_{S}\right) .
$$

Here and after, dim ${ }_{\mathcal{H}} E$ denotes the Hausdorff dimension of the set $E$.

Let us comment the proof. To get the upper bound, we combine the classical variation methods with the "slicing" technique introduced in [25], which allows to distinguish different local behavior of $M$, see Sect. 3. To get the lower bound, the strategy is to couple our process with a family of other stable-like processes whose Hausdorff dimension is known and then compare the Hausdorff dimension of their sample paths with ours using pathwise uniqueness and the Markov property, see Sect. 4. 
With the same strategy, we are also able to compute the Hausdorff dimension of the graph of stable-like processes.

Theorem 2 Let $M$ be a stable-like process as in Theorem 1. Let $\operatorname{Gr}_{I}(M)=\left\{\left(t, M_{t}\right)\right.$ : $t \in I\}$ be the graph of $M$ on the interval $I \subset \mathbb{R}^{+}$.

1. If $d \geq 2$, then a.s. for every open interval $I \subset \mathbb{R}^{+}$,

$$
\operatorname{dim}_{\mathcal{H}}\left(\operatorname{Gr}_{I}(M)\right)=1 \vee \sup _{t \in I} \beta\left(M_{t}\right)
$$

2. If $d=1$, then a.s. for every open interval $I \subset \mathbb{R}^{+}$,

$$
\operatorname{dim}_{\mathcal{H}}\left(G r_{I}(M)\right)=1 \vee\left(2-\frac{1}{\sup _{t \in I} \beta\left(M_{t}\right)}\right)
$$

This theorem generalizes classical results $[3,8]$ on the Hausdorff dimension for the graph of $\alpha$-stable processes in $\mathbb{R}^{d}$. Historically, Blumenthal and Getoor [3] treated the recurrent case $(d=1$ and $\alpha>1)$, and Jain and Pruitt [8] the transient case $(\alpha<d)$. Later, Pruitt and Taylor [16] investigated, among others things, the asymptotic behavior of the sojourn time of a Lévy process with stable components and related the exact Hausdorff measure of the graph of such process to these results. We follow and adapt, when necessary, the arguments of Pruitt and Taylor [16].

This paper is organized as follows. We first recall some basic properties of the stable-like processes in Sect. 2. We study the $p$-variation of $M$ in Sect. 3 to yield the upper bound for the dimension of the range of stable-like processes. The lower bound is proved in Sect. 4 using a coupling argument. Finally, we deal with the dimension of the graph of $M$ (Theorem 2) in Sect. 5.

In the whole paper, $C$ is a positive finite constant independent of the problem that may change from line to line.

From now on, we only consider the time interval $[0,1]$; extension to any interval is straightforward.

\section{Preliminaries}

First let us introduce the SDE with jumps associated with stable-like processes. Let $\left(\Omega, \mathcal{F},\left(\mathcal{F}_{t}\right), \mathbb{P}\right)$ be a filtered probability space satisfying the usual conditions. Let $\lambda$ be the Lebesgue measure on $\mathbb{R}^{+}, H$ be the uniform probability measure on $\mathbb{S}^{d-1}$ and $\pi(d r)=r^{-2} \mathrm{~d} r$ on $\mathbb{R}^{+}$. Denote by $N$ a Poisson random measure on the product space $\mathbb{R}^{+} \times \mathbb{S}^{d-1} \times \mathbb{R}^{+}$adapted to the filtration $\left(\mathcal{F}_{t}\right)$ and with intensity $\lambda \otimes H \otimes \pi$. We denote by $\widetilde{N}$ the corresponding compensated Poisson measure.

Proposition 1 Let $\beta$ be as in (1). For every $\mathcal{F}_{0}$-measurable random variable $M_{0}$, there exists a unique pathwise solution to the stochastic differential equation, 


$$
\begin{aligned}
M_{t}= & M_{0}+\int_{0}^{t} \int_{S^{d-1}} \int_{0}^{1} \theta r^{1 / \beta\left(M_{s-}\right)} \tilde{N}(\mathrm{~d} s, \mathrm{~d} \theta, \mathrm{d} r) \\
& +\int_{0}^{t} \int_{\mathbb{S}^{d}} \int_{1}^{+\infty} \theta r^{1 / \beta\left(M_{s-}\right)} N(\mathrm{~d} s, \mathrm{~d} \theta, \mathrm{d} r) .
\end{aligned}
$$

Furthermore, the solution to $(3)$ is a càdlàg $\left(\mathcal{F}_{t}\right)$-adapted Feller process whose generator is $\mathcal{L}^{\beta}$ in (1).

Remark 1 This SDE representation for stable-like processes was first proved in [23, page 111]; we include a proof for completeness.

Proof Let us first consider the well-posedness of the SDE. By an interlacement procedure for the noncompensated Poisson integral in (3) (see [6, Proposition 2.4]), it is enough to prove that the following SDE has a unique pathwise solution:

$$
X_{t}=X_{0}+\int_{0}^{t} \int_{S^{d-1}} \int_{0}^{1} \theta r^{1 / \beta\left(X_{s-}\right)} \tilde{N}(\mathrm{~d} s, \mathrm{~d} \theta, \mathrm{d} r)
$$

Classical Picard iteration, Gronwall's lemma and localization procedure entail the existence of a unique pathwise solution once we check the usual (local) Lipschitz continuity and linear growth condition on the coefficients of the SDE, see for instance [20, Section 3.1]. In other words, it suffices to check that there exists a positive finite constant $C$ such that for all $x, y \in \mathbb{R}^{d}$,

$$
\begin{array}{r}
\int_{\mathbb{S}^{d-1}} \int_{0}^{1}\left|\theta r^{1 / \beta(x)}\right|^{2} r^{-2} \mathrm{~d} r H(\mathrm{~d} \theta) \leq C\left(1+|x|^{2}\right), \\
\int_{\mathbb{S}^{d-1}} \int_{0}^{1}\left|\theta r^{1 / \beta(x)}-\theta r^{1 / \beta(y)}\right|^{2} r^{-2} \mathrm{~d} r H(\mathrm{~d} \theta) \leq C|x-y|^{2} .
\end{array}
$$

Actually, the first integral is bounded from above uniformly in $x$, that is

$$
\int_{\mathbb{S}^{d-1}} \int_{0}^{1}\left|\theta r^{1 / \beta(x)}\right|^{2} r^{-2} \mathrm{~d} r H(\mathrm{~d} \theta) \leq C
$$

These conditions are checked in Appendix.

To prove the second statement, one starts with the observation that

$$
\mathcal{L}^{\beta} f(x)=\int_{\mathbb{S}^{d-1}} \int_{\mathbb{R}^{+}}\left(f\left(x+\theta r^{1 / \beta(x)}\right)-f(x)-1_{0<r<1} r^{1 / \beta(x)} \theta \cdot \nabla f(x)\right) \frac{\mathrm{d} r}{r^{2}} H(\mathrm{~d} \theta),
$$

where a change of variable $u=\theta r^{1 / \beta(x)}$ was used for all $x \in \mathbb{R}^{d}$ in (1). Applying Itô's formula to $M$ the solution of (3), for all $f \in C_{c}^{2}\left(\mathbb{R}^{d}\right)$, 


$$
\begin{aligned}
& f\left(M_{t}\right)-f\left(M_{0}\right)-\int_{0}^{t} \mathcal{L}^{\beta} f\left(M_{S}\right) \mathrm{d} s \\
& =\int_{0}^{t} \int_{\mathbb{S}^{d-1}} \int_{0}^{1}\left(f\left(M_{s-}+\theta r^{1 / \beta\left(M_{s-}\right)}\right)-f\left(M_{s-}\right)\right) \tilde{N}(\mathrm{~d} s, \mathrm{~d} \theta, \mathrm{d} r) \\
& \quad+\int_{0}^{t} \int_{\mathbb{S}^{d-1}} \int_{1}^{+\infty}\left(f\left(M_{s-}+\theta r^{1 / \beta\left(M_{s-}\right)}\right)-f\left(M_{s-}\right)\right) \tilde{N}(\mathrm{~d} s, \mathrm{~d} \theta, \mathrm{d} r) .
\end{aligned}
$$

Applying mean value theorem to $f$ (around zero) and (5) for the first compensated Poisson integral, then $\|f\|_{\infty}<+\infty$ for the second, one concludes that

$$
f\left(M_{t}\right)-f\left(M_{0}\right)-\int_{0}^{t} \mathcal{L}^{\beta} f\left(M_{s}\right) \mathrm{d} s
$$

is a martingale. By the uniqueness of the martingale problem for $\left(\mathcal{L}^{\beta}, C_{c}^{2}\left(\mathbb{R}^{d}\right)\right)$ due to Bass [1], one concludes that the solution to (3) is a stable-like process with index function $\beta$. The Feller property was proved in [1, page 285]. The proof is now complete.

An application of (5) and Burkholder-Davis-Gundy's inequality yields the following fact.

Lemma 1 The compensated Poisson integral in (3) is a martingale in $L^{2}(\Omega)$.

We also need to compute the symbol of the operator $\mathcal{L}^{\beta}$ in order to use known dimension bounds for the range of Lévy-type processes.

Lemma 2 The domain of $\mathcal{L}^{\beta}$ contains $C_{c}^{\infty}\left(\mathbb{R}^{d}\right)$ the space of smooth functions with compact support and the restriction of $\mathcal{L}^{\beta}$ on $C_{c}^{\infty}\left(\mathbb{R}^{d}\right)$ is a pseudo-differential operator with symbol $q(x, \xi)=a(x)|\xi|^{\beta(x)}$ with $a: \mathbb{R}^{d} \rightarrow \mathbb{R}$ continuous bounded below and above by two positive finite constants.

Proof The first statement is obvious. It remains to compute the symbol. Set

$$
\mathcal{F} f(\xi)=\int f(x) e^{i x \cdot \xi} \mathrm{d} x
$$

For all $f \in C_{c}^{\infty}\left(\mathbb{R}^{d}\right)$, by Fubini's theorem, $\mathcal{F}\left(\mathcal{L}^{\beta} f\right)(\xi)=-q(x, \xi) \mathcal{F} f(\xi)$ where

$$
q(x, \xi)=\int\left(1-e^{-i u \cdot \xi}-i \xi \cdot u 1_{|u| \leq 1}\right) u^{-d-\beta(x)} \mathrm{d} u
$$

Set for $\alpha \in(0,2)$,

$$
C_{\alpha}=\int_{0}^{\infty}(1-\cos r) r^{-1-\alpha} \mathrm{d} r .
$$

Using spherical coordinate and by symmetry, 


$$
\begin{aligned}
q(x, \xi) & =\int_{\mathbb{S}^{d-1}} \int_{0}^{\infty}\left(1-e^{i r(\theta \cdot \xi)}-i(\theta \cdot \xi) r 1_{r<1}\right) r^{-1-\beta(x)} \mathrm{d} r \mathrm{~d} \theta \\
& =\int_{\mathbb{S}^{d-1}} \int_{0}^{\infty}(1-\cos (r(\theta \cdot \xi))) r^{-1-\beta(x)} \mathrm{d} r \mathrm{~d} \theta \\
& =C_{\beta(x)} \int_{\mathbb{S}^{d-1}}|\theta \cdot \xi|^{\beta(x)} \mathrm{d} \theta \\
& =a(x)|\xi|^{\beta(x)}
\end{aligned}
$$

where

$$
a(x)=C_{\beta(x)} \int_{\mathbb{S}^{d-1}}\left|\theta \cdot \mathbf{e}_{1}\right|^{\beta(x)} \mathrm{d} \theta \quad \text { with } \mathbf{e}_{1}=(1,0, \ldots, 0) \in \mathbb{R}^{d}
$$

This completes the proof.

For stable-like processes with symbol as in previous lemma, Kolokoltsov [12, Chapter 7] (see also [11]) showed the existence of the transition densities and provided fine heat kernel estimates. Let us recall the part that is useful for our purposes.

Lemma 3 ([12]) Let $p(t, x, y)$ be the transition density of stable-like processes with index $\beta$. Let $\alpha=\inf _{x \in \mathbb{R}^{d}} \beta(x)$. Then there exists a finite positive $C$ so that $t<$ $1, x, y \in \mathbb{R}^{d}$,

$$
p(t, x, y) \leq C t^{-d / \alpha} \text {. }
$$

Let us end this section with known dimension estimates for the range of Lévy-type processes.

Lemma $4([10,18])$ Let $\left(X_{t}\right)_{t \geq 0}$ be a Feller process with generator $(A, \mathcal{D}(A))$ such that $A_{\mid C_{c}^{\infty}\left(\mathbb{R}^{d}\right)}$ is a pseudo-differential operator with symbol $q(x, \xi)$ satisfying $|q(x, \xi)| \leq c\left(1+|\xi|^{2}\right)$ for all $x$ and $q(\cdot, 0) \equiv 0$. Then almost surely, $\operatorname{dim}_{\mathcal{H}}(X[0,1]) \leq d \wedge \beta_{\infty}$ where

$$
\beta_{\infty}=\inf \left\{\delta>0: \lim _{|\xi| \rightarrow \infty} \frac{\sup _{|\eta| \leq|\xi|} \sup _{x \in \mathbb{R}^{d}}|q(x, \eta)|}{|\xi|^{\delta}}=0\right\}
$$

If in addition the transition density of the process $X$ exists and satisfies (6) for some constants $C$ and $\alpha \in(0,2)$, then

$$
\operatorname{dim}_{\mathcal{H}}(X[0,1]) \geq d \wedge \alpha \quad \text { a.s. }
$$

Combining previous lemmas, it is now plain that the Hausdorff dimension for the range of stable-like processes is bounded above by $\sup _{x \in \mathbb{R}^{d}} \beta(x) \wedge d$ and bounded below by $\inf _{x \in \mathbb{R}^{d}} \beta(x) \wedge d$. We prove in the sequel that neither bound is optimal. 


\section{Study of the $p$-variation of $M$ : upper bound of Theorem 1}

The aim of this section is to prove that

$$
\operatorname{dim}_{\mathcal{H}}(M([0,1])) \leq \beta_{M}^{*} \wedge d, \text { where } \beta_{M}^{*}=\sup _{t \in[0,1]} \beta\left(M_{t}\right) .
$$

We use a slicing procedure for $M$ and the $p$-variation approach to tackle this problem. The use of $p$-variation in deducing an upper bound for the Hausdorff dimension of the range of sample paths goes back, at least, to McKean [14]. In this article we apply a theorem by Lépingle [13] on the $p$-variation of semimartingales.

First let us introduce some notations for the $p$-variation of functions.

Let $f: \mathbb{R}^{+} \rightarrow \mathbb{R}^{d}$ be a càdlàg function and $\mathcal{P}$ be a finite partition of the interval $[0, t]$ deduced naturally from a family of strictly ordered points $\left(0=t_{0}<\cdots<t_{n}=t\right)$. Following the notations in [13], for any $p \in(0,2)$, let

$$
V_{p}(f, \mathcal{P})=\sum_{i=0}^{n-1}\left|f\left(t_{i+1}\right)-f\left(t_{i}\right)\right|^{p}
$$

Then the (strong) $p$-variation of $f$ in the interval $[0, t]$ is

$$
W_{p}(f,[0, t])=\sup \left\{V_{p}(f, \mathcal{P}): \mathcal{P} \text { finite partition of }[0, t]\right\} .
$$

We also introduce the quantity corresponding to the jumps of $f$ in the interval $[0, t]$,

$$
S_{p}(f,[0, t])=\sum_{0<s \leq t}\left|\Delta f_{s}\right|^{p},
$$

where $\Delta f_{s}=f(s)-f(s-)$ and $f(s-)=\lim _{t \uparrow s} f(t)$.

Recall that a semimartingale is a process of the form $X_{t}=X_{0}+M_{t}+A_{t}$, where $X_{0}$ is finite a.s. and is $\mathcal{F}_{0}$ measurable, $M_{t}$ is a local martingale, and $A_{t}$ is a process whose sample paths have bounded variation on $[0, t]$ for each $t$. Such a process can be written as $X_{t}=X_{t}^{c}+X_{t}^{j}$, the sum of a continuous part $X^{c}$ and a pure jump part $X^{j}$. Let us state a part of Lépingle's result (see Theorem 1 of [13]) which is useful for our purpose.

Theorem 3 ([13]) Let $X$ be a semimartingale such that $\left\langle X^{c}\right\rangle$. $\equiv 0$. Let $p>0$. Then almost surely,

$$
S_{p}(X,[0,1])<+\infty \Longrightarrow\left(\forall p^{\prime}>p, W_{p^{\prime}}(X,[0,1])<+\infty\right) \text {. }
$$

Following [25], we slice the process $M$ according to the different behavior of the local index process $t \mapsto \beta\left(M_{t}\right)$. This induces a decomposition for the process $M$. Precisely, for every $m \in \mathbb{N}_{*}$, we write $M .=M_{0}+\sum_{k=0}^{m-1} M^{k, m}+M^{\geq 1}$ where

$$
M_{t}^{k, m}=\int_{0}^{t} \int_{S^{d-1}} \int_{0}^{1} \theta r^{1 / \beta\left(M_{s-}\right)} 1_{\beta\left(M_{s-}\right) \in\left[\frac{2 k}{m}, \frac{2 k+2}{m}\right)} \tilde{N}(\mathrm{~d} s, \mathrm{~d} \theta, \mathrm{d} r) .
$$


and

$$
M_{t}^{\geq 1}=\int_{0}^{t} \int_{\mathbb{S}^{d}} \int_{1}^{+\infty} \theta r^{1 / \beta\left(M_{s-}\right)} N(\mathrm{~d} s, \mathrm{~d} \theta, \mathrm{d} r) .
$$

From a trajectory point of view, each sliced process behaves exactly the same as $M$ when the index process takes value in the sliced interval; otherwise, it is only a constant process. The process $M^{\geq 1}$ is not relevant in the computation of $p$-variation for $M$ since it is piecewise constant with finite number of jumps in the unit interval.

Now we are ready to prove (8). For each $(k, m)$, we study the $p$-variation of $M^{k, m}$ and then deduce the finiteness of the $p$-variation of the whole process $M$ for any $p>\beta_{M}^{*}$. The desired inequality follows by a general argument by McKean [14] on the relation between Hausdorff dimension of the range of a function and its $p$-variation.

Lemma 5 For every $m \in \mathbb{N}_{*}$ and every $k=0, \ldots, m$, almost surely,

$$
W_{\frac{2 k+3}{m}}\left(M^{k, m},[0,1]\right)<+\infty .
$$

Proof The method consists in applying Theorem 3 to each $M^{k, m}$ since each of them is a semimartingale satisfying $\left\langle\left(M^{k, m}\right)^{c}\right\rangle$. $\equiv 0$. We start with the observation that

$$
\begin{aligned}
& S_{\left(2 k+\frac{5}{2}\right) / m}\left(M^{k, m},[0,1]\right) \\
& \quad=\int_{0}^{1} \int_{\mathbb{S}^{d-1}} \int_{0}^{1} r^{\left(2 k+\frac{5}{2}\right) / m \beta\left(M_{s-}\right)} 1_{\beta\left(M_{s-}\right) \in[2 k / m,(2 k+2) / m)} N(\mathrm{~d} s, \mathrm{~d} \theta, \mathrm{d} r) .
\end{aligned}
$$

Taking expectation, we see that

$$
\begin{aligned}
\mathbb{E} & {\left[S_{\left(2 k+\frac{5}{2}\right) / m}\left(M^{k, m},[0,1]\right)\right] } \\
& =\int_{0}^{1} \int_{0}^{1} \mathbb{E}\left[r^{\left(2 k+\frac{5}{2}\right) / m \beta\left(M_{s-}\right)} 1_{\beta\left(M_{s-}\right) \in[2 k / m,(2 k+2) / m)}\right] \mathrm{d} s \frac{\mathrm{d} r}{r^{2}} \\
& \leq \int_{0}^{1} \int_{0}^{1} r^{\left(2 k+\frac{5}{2}\right) /(2 k+2)-2} \mathrm{~d} r \mathrm{~d} s<+\infty .
\end{aligned}
$$

Consequently, $S_{\left(2 k+\frac{5}{2}\right) / m}\left(M^{k, m},[0,1]\right)$ is finite almost surely. An application of Theorem 3 ends the proof.

Now the finiteness of the $p$-variation of the whole process $M$ is proved as follows.

Lemma 6 Almost surely, for any $p>\beta_{M}^{*}$,

$$
W_{p}(M,[0,1])<+\infty .
$$

Proof Recall that $\beta_{M}^{*}$ is defined in (8). Consider the events

$$
A_{k, m}=\left\{\beta_{M}^{*}+\frac{3}{m} \geq \frac{2 k+3}{m}\right\}, \quad B_{k, m}=\left\{W_{\beta_{M}^{*}+\frac{3}{m}}\left(M^{k, m},[0,1]\right)<+\infty\right\} .
$$


Since the mapping $p \mapsto 1_{W_{p}}(f,[0,1])<\infty$ is nondecreasing, one has

$$
\mathbb{P}\left(B_{k, m} \cap A_{k, m}\right) \geq \mathbb{P}\left(\left\{W_{\frac{2 k+3}{m}}\left(M^{k, m},[0,1]\right)<+\infty\right\} \cap A_{k, m}\right) .
$$

Under $A_{k, m}^{c}$, i.e., the complementary of $A_{k, m}, M^{k, m} \equiv 0$ by the properties of the compensated Poisson integral. Hence $B_{k, m}$ is also realized. This inclusion $A_{k, m}^{c} \subset$ $B_{k, m}$ yields

$$
\mathbb{P}\left(B_{k, m} \cap A_{k, m}^{c}\right)=\mathbb{P}\left(A_{k, m}^{c}\right)
$$

Combining the previous two estimates, one obtains

$$
\begin{aligned}
\mathbb{P}\left(B_{k, m}\right) & =\mathbb{P}\left(B_{k, m} \cap A_{k, m}\right)+\mathbb{P}\left(B_{k, m} \cap A_{k, m}^{c}\right) \\
& \geq \mathbb{P}\left(\left\{W_{\frac{2 k+3}{m}}\left(M^{k, m},[0,1]\right)<+\infty\right\} \cap A_{k, m}\right)+\mathbb{P}\left(A_{k, m}^{c}\right) \\
& \geq \mathbb{P}\left(W_{\frac{2 k+3}{m}}\left(M^{k, m},[0,1]\right)<+\infty\right)=1,
\end{aligned}
$$

where Lemma 5 has been used. By Jensen's inequality (when $p \geq 1$ ) or subadditivity (when $p<1$ ), for any $p \in(0,3), n \in \mathbb{N}_{*}$ and $\left(a_{1}, \ldots, a_{n}\right) \in \mathbb{R}^{n}$, one has $\left(\sum_{i=1}^{n}\left|a_{i}\right|\right)^{p} \leq\left(n^{p-1} \vee 1\right) \sum_{i=1}^{n}\left|a_{i}\right|^{p}$. This yields for any finite partition, every family of càdlàg functions $f_{i}:[0,1] \rightarrow \mathbb{R}$ with $i=1, \ldots, n$ that

$$
V_{p}\left(\sum_{i=1}^{n} f_{i}, \mathcal{P}\right) \leq C(n, p) \sum_{i=1}^{n} V_{p}\left(f_{i}, \mathcal{P}\right) \leq C(n, p) \sum_{i=1}^{n} W_{p}\left(f_{i},[0,1]\right),
$$

where $C(n, p)=n^{p-1} \vee 1$. Therefore, since $\mathbb{P}\left(B_{k, m}\right)=1$, one has a.s.

$$
W_{\beta_{M}^{*}+\frac{3}{m}}(M,[0,1]) \leq C\left(m, \beta_{M}^{*}+\frac{3}{m}\right) \sum_{k=1}^{m} W_{\beta_{M}^{*}+\frac{3}{m}}\left(M^{k, m},[0,1]\right)<+\infty
$$

for every $m \in \mathbb{N}_{*}$, which yields the result.

Proof of Formula (8) Recall the following fact in [14]: if $f:[0,1] \rightarrow \mathbb{R}^{d}$ is a càdlàg function with finite $p$-variation, then

$$
\operatorname{dim}_{\mathcal{H}}(f[0,1]) \leq p \wedge d
$$

Now (8) follows by combining the fact above and Lemma 6.

\section{Lower Bound of Theorem 1}

Throughout this section, we use $\mathbb{P}^{x}$ to denote the law of $M$ with initial value $M_{0}=$ $x \in \mathbb{R}^{d}$. Denote by $\mathbb{E}^{x}$ the expectation with respect to $\mathbb{P}^{x}$. 
To prove the lower bound, we introduce a suitable coupling of $M$ with a family of processes whose dimension of the range is known. This coupling is used in the proof of the following lemma; see (10) below.

Lemma 7 Let $0 \leq t_{0}<1$. For every $z \in \mathbb{R}^{d}, \mathbb{P}^{z}$-a.s.

$$
\operatorname{dim}_{\mathcal{H}}\left(M\left[t_{0}, 1\right]\right) \geq \beta\left(M_{t_{0}}\right) \wedge d
$$

Proof For any $z \in \mathbb{R}^{d}$, by the Markov property,

$$
\mathbb{P}^{z}\left(\operatorname{dim}_{\mathcal{H}}\left(M\left[t_{0}, 1\right]\right) \geq \beta\left(M_{t_{0}}\right) \mid \mathcal{F}_{t_{0}}\right)=g\left(M_{t_{0}}\right) \text { a.s. }
$$

where

$$
g(x)=\mathbb{P}^{x}\left(\operatorname{dim}_{\mathcal{H}}\left(M\left[0,1-t_{0}\right]\right) \geq \beta(x)\right) .
$$

Now one constructs a coupling with the process $M$. Let $a \in(0,2), x \in \mathbb{R}^{d}$ and $\beta_{a}(\cdot)=\beta(\cdot) \vee a$. For each $\varepsilon>0$ and any rational number $0<a \leq \beta(x)-2 \varepsilon$, one introduces the process $M^{x, a}$, solution to the SDE

$$
\begin{aligned}
M_{t}^{x, a}= & x+\int_{0}^{t} \int_{S^{d-1}} \int_{0}^{1} \theta r^{1 / \beta_{a}\left(M_{s-}^{x, a}\right)} \tilde{N}(\mathrm{~d} s, \mathrm{~d} \theta, \mathrm{d} r) \\
& +\int_{0}^{t} \int_{\mathbb{S}^{d}} \int_{1}^{+\infty} \theta r^{1 / \beta_{a}\left(M_{s-}\right)} N(\mathrm{~d} s, \mathrm{~d} \theta, \mathrm{d} r)
\end{aligned}
$$

driven by the same Poisson random measure. Existence and pathwise uniqueness of these processes can be proved as in Proposition 1.

Define the stopping times

$$
\begin{aligned}
\tau_{x} & =\inf \left\{t \geq 0: \beta\left(M_{t}\right) \leq \beta(x)-\varepsilon\right\}, \\
\tau_{x, a} & =\inf \left\{t \geq 0: \beta\left(M_{t}^{x, a}\right) \leq \beta(x)-\varepsilon\right\} .
\end{aligned}
$$

Define also

$$
\tau_{\geq 1}=\inf \{0 \leq t \leq 1: N([0, t] \times[1,+\infty)) \geq 1\}
$$

By the càdlàg property of the sample paths of $M^{x, a}$ and $M$, all these stopping times are strictly positive $\mathbb{P}^{x}$-a.s. Note that $\tau_{\geq 1}$ is an exponential random variable with finite parameter, it is also strictly positive $\mathbb{P}^{x}$ almost surely. Set $\tau=\min \left(\tau_{x}, \tau_{x, a}, \tau_{\geq 1}\right) / 2$. The following observation is fundamental :

$$
\mathbb{P}^{x} \text { a.s. } \quad \forall t \geq 0, \quad M_{t \wedge \tau}=M_{t \wedge \tau}^{x, a} .
$$


Indeed, for every $t \geq 0$, using $\tau<\tau_{\geq 1}$, one remarks that the large jump term is identically zero before time $\tau$ so that

$$
\begin{aligned}
\mathbb{E}^{x}\left[\left|M_{t \wedge \tau}^{x, a}-M_{t \wedge \tau}\right|^{2}\right] \\
\quad=\mathbb{E}\left[\left|\int_{0}^{t \wedge \tau} \int_{S^{d-1}} \int_{0}^{1} \theta\left(r^{1 / \beta_{a}\left(M_{s-}^{x, a}\right)}-r^{1 / \beta\left(M_{s-}\right)}\right) \tilde{N}(\mathrm{~d} s \mathrm{~d} \theta \mathrm{d} r)\right|^{2}\right] .
\end{aligned}
$$

By Burkholder-Davis-Gundy inequality and Lipschitz continuity of $\beta$,

$$
\begin{aligned}
& \mathbb{E}^{x} {\left[\left|M_{t \wedge \tau}^{x, a}-M_{t \wedge \tau}\right|^{2}\right] } \\
& \leq C \mathbb{E}^{x}\left[\int_{0}^{t \wedge \tau} \int_{S^{d-1}} \int_{0}^{1}\left|r^{1 / \beta_{a}\left(M_{s-}^{x, a}\right)}-r^{1 / \beta\left(M_{s-}\right)}\right|^{2} \frac{\mathrm{d} r}{r^{2}} H(\mathrm{~d} \theta) \mathrm{d} s\right] \\
&=C \mathbb{E}^{x}\left[\int_{0}^{t} \int_{0}^{1}\left|r^{1 / \beta\left(M_{s-\wedge \tau}^{x, a}\right)}-r^{1 / \beta\left(M_{s-\wedge \tau}\right)}\right|^{2} \frac{\mathrm{d} r}{r^{2}} \mathrm{~d} s\right] \\
& \leq C \mathbb{E}^{x}\left[\int_{0}^{t}\left|M_{s \wedge \tau}^{x, a}-M_{s \wedge \tau}\right|^{2} \mathrm{~d} s\right] \\
&=C \int_{0}^{t} \mathbb{E}^{x}\left[\left|M_{s \wedge \tau}^{x, a}-M_{s \wedge \tau}\right|^{2}\right] \mathrm{d} s,
\end{aligned}
$$

Hence, using Gronwall's lemma, for every $t \geq 0$,

$$
\mathbb{E}^{x}\left[\left|M_{t \wedge \tau}^{a}-M_{t \wedge \tau}\right|^{2}\right]=0
$$

This, along with the càdlàg property of the sample paths, yields (11).

To conclude, applying Lemma 4 (lower bound) to the stable-like process $M^{x, a}$ with index function $\beta_{a}$, we obtain that for each $t \in(0,1]$,

$$
\mathbb{P}^{x} \text { a.s. } \quad \operatorname{dim}_{\mathcal{H}} M^{x, a}([0, t]) \geq \inf _{x \in \mathbb{R}^{d}} \beta_{a}(x) \wedge d \geq a \wedge d .
$$

This full probability set is indexed by $t$ and is nondecreasing as $t$ increases. Hence almost surely, for all $t \in(0,1]$ and all rational $a \in(1, \beta(x)-2 \varepsilon)$, one has $\operatorname{dim}_{\mathcal{H}} M^{x, a}([0, t]) \geq a \wedge d$. One deduces that $\mathbb{P}^{x}$ a.s.

$$
\begin{aligned}
& \operatorname{dim}_{\mathcal{H}} M\left(\left[0,1-t_{0}\right]\right) \geq \operatorname{dim}_{\mathcal{H}} M\left(\left[0, \tau \wedge\left(1-t_{0}\right)\right]\right) \\
& \quad=\operatorname{dim}_{\mathcal{H}} M^{x, a}\left(\left[0, \tau \wedge\left(1-t_{0}\right)\right]\right) \geq a \wedge d,
\end{aligned}
$$

where we used (11) for the equality and the fact that $\mathbb{P}^{x}$ a.s. $\tau>0$ for the last inequality. Letting $a \rightarrow \beta(x)-2 \varepsilon$ along a countable sequence, then letting $\varepsilon \rightarrow 0$, one obtains that

$$
g(x) \equiv 1
$$


One concludes with $\mathbb{P}^{z}\left(\operatorname{dim}_{\mathcal{H}} M\left(\left[t_{0}, 1\right]\right) \geq \beta\left(M_{t_{0}}\right)\right)=\mathbb{E}^{z}\left[g\left(M_{t_{0}}\right)\right]=1$. This completes the proof.

Finally, we prove the lower bound in Theorem 1.

Proof Using Lemma 7, we have for each $t_{0} \in[0,1)$ that $\mathbb{P}^{z}$ a.s.

$$
\operatorname{dim}_{\mathcal{H}}(M([0,1])) \geq \operatorname{dim}_{\mathcal{H}}\left(M\left(\left[t_{0}, 1\right]\right)\right) \geq \beta\left(M_{t_{0}}\right) \wedge d
$$

then $\mathbb{P}^{z}$ a.s.

$$
\operatorname{dim}_{\mathcal{H}}(M([0,1])) \geq \sup _{t_{0} \in[0,1) \cap \mathbb{Q}} \beta\left(M_{t_{0}}\right) \wedge d=\sup _{t_{0} \in[0,1]} \beta\left(M_{t_{0}}\right) \wedge d,
$$

where we used the càdlàg property of the sample paths. Since this holds for any $z \in \mathbb{R}^{d}$, one deduce that the lower bound holds for any measurable $M_{0} \in \mathcal{F}_{0}$.

\section{Dimension of the Graph of $M$ : proof of Theorem 2}

\subsection{Case $d \geq 2$}

Since a projection never increases the dimension of a subset of $\mathbb{R}^{d}$, projecting the graph on the time axis and then on the space axis yields the announced lower bound for dimension of the graph.

It remains us to prove the other inequality. Recall that $\beta_{M}^{*}=\sup _{t \in[0,1]} \beta\left(M_{t}\right)$. For every $p>\max \left(1, \beta_{M}^{*}\right) \geq \beta_{M}^{*}=\beta_{M}^{*} \wedge d$, consider the $p$-variation of the process $\mathcal{G}(t)=\left(\operatorname{Id}(t), M_{t}\right)$ in $\mathbb{R}^{d+1}$, where $\operatorname{Id}(t)=t$. As $W_{p}(\mathrm{Id},[0,1]) \leq 1$ for every $p>1$, there exists a constant $C=C(d)$ such that

$$
W_{p}(\mathcal{G},[0,1]) \leq C\left(1+W_{p}(M,[0,1])\right)<+\infty
$$

by Lemma 6. Applying (9) yields the desired upper bound.

\subsection{Case $d=1$}

The proof is split into several parts. The first one gives an upper bound for the upper box-counting dimension of the graph of $M$, which in turn gives an upper bound for the Hausdorff dimension of the graph. Recall that the upper box-counting dimension of a set $E \subset \mathbb{R}^{d}$ is defined by

$$
\overline{\operatorname{dim}_{B}}(E)=\limsup _{\delta \downarrow 0} \frac{\log N_{\delta}(E)}{-\log \delta},
$$

where $N_{\delta}(E)$ is the smallest number of sets of diameter at most $\delta$ to cover $E$, see Chapter 3 of [5]. The proof is quite standard, see for instance [15]. We prove it for completeness. 


\section{Proposition 2 Almost surely,}

$$
\operatorname{dim}_{\mathcal{H}}\left(\operatorname{Gr}_{[0,1]}(M)\right) \leq \overline{\operatorname{dim}_{B}}\left(\operatorname{Gr}_{[0,1]}(M)\right) \leq \max \left(1,2-\frac{1}{\beta_{M}^{*}}\right)
$$

Proof The left inequality is a general fact, see [5, page 46]. Let us prove the right inequality. If the event $\left\{\beta_{M}^{*}<1\right\}$ is realized, Lemma 6 yields that the process $M$ has finite variation; a fortiori, the graph process $\mathcal{G}$ has finite variation. Hence the dimension of the graph of $M$ (which is the range of $\mathcal{G}$ ) is 1 by the projection argument used in Sect. 5.1 and (9). The desired inequality is straightforward.

If $\left\{\beta_{M}^{*} \geq 1\right\}$ is realized, we consider $p>\beta_{M}^{*} \geq 1$ and relate the upper box dimension with the $p$-variation of the process. Denote the oscillation of the process $M$ in the dyadic interval $\left[k 2^{-j},(k+1) 2^{-j}\right]$ by

$$
\operatorname{Osc}\left(M, I_{j, k}\right):=\sup \left\{\left|M_{s}-M_{t}\right|: s, t \in I_{j, k}\right\} .
$$

For every $k, G r_{I_{j, k}}(M)$ can be covered by at most $2^{j} O s c\left(M, I_{j, k}\right)+2$ squares of side length $2^{-j}$. The number of squares of generation $j$ required to cover the graph $\operatorname{Gr}_{[0,1]}(M)$ satisfies

$$
\begin{aligned}
N_{j} & =\sum_{k=0}^{2^{j}-1}\left(2^{j} \operatorname{Osc}\left(M, I_{j, k}\right)+2\right) \leq 2^{j} \sum_{k=0}^{2^{j}-1} W_{p}\left(M, I_{j, k}\right)^{\frac{1}{p}}+2 \cdot 2^{j} \\
& \leq 2^{j}\left(\sum_{k=0}^{2^{j}-1} W_{p}\left(M, I_{j, k}\right)\right)^{\frac{1}{p}}\left(2^{j}\right)^{1-\frac{1}{p}}+2 \cdot 2^{j} \\
& \leq 2 \cdot 2^{j\left(2-\frac{1}{p}\right)} W_{p}(M,[0,1])^{\frac{1}{p}}
\end{aligned}
$$

for all $j$ large enough, where we used Hölder inequality for the second inequality. Therefore,

$$
\overline{\operatorname{dim}_{B}}\left(G r_{[0,1]}(M)\right) \leq \limsup _{j \rightarrow \infty} \frac{\log N_{j}}{\log 2^{j}} \leq 2-\frac{1}{p}
$$

where we used $W_{p}(M,[0,1])<\infty$. Letting $p \rightarrow \beta_{M}^{*}$ yields the result.

The rest of this section is devoted to prove the lower bound in Theorem 2,

$$
\operatorname{dim}_{\mathcal{H}}\left(\operatorname{Gr}_{[0,1]}(M)\right) \geq 1 \vee\left(2-\frac{1}{\beta_{M}^{*}}\right)
$$

To prove it, we give a deterministic lower bound for the dimension of graph. This should be viewed as an analogue of Lemma 4 (the lower bound part) in the graph context. 
Proposition 3 Denote $\underline{\beta}=\inf _{x \in \mathbb{R}^{d}} \beta(x)$. Almost surely,

$$
\operatorname{dim}_{\mathcal{H}}\left(\operatorname{Gr}_{[0,1]}(M)\right) \geq 1 \vee\left(2-\frac{1}{\underline{\beta}}\right) .
$$

We prove this proposition in several steps. First we adapt the ideas in [16] to give tail estimates for the sojourn time of $M$. This allows to understand the local behavior of the graph occupation measure. Then we use a density argument to obtain the lower bound for the Hausdorff dimension of the graph of $M$.

Following Pruitt-Taylor [16], we define the sojourn time of $M$ in the ball centered at $M_{t_{0}}$ with radius $a>0$, during the time interval $\left[t_{0}, t_{0}+s\right]$ for $0<s<1-t_{0}$ as

$$
T_{t_{0}}(a, s)=\int_{t_{0}}^{t_{0}+s} 1_{\mid M_{t}-M_{t_{0} \mid \leq a} d t .}
$$

Write for simplicity $T(a, s)=T_{0}(a, s)$. The main estimate is the following.

Lemma 8 Fix $t_{0} \in[0,1)$. Assume that $\underline{\beta}>1$ and let $C=\frac{2}{1-1 / \underline{\beta}}$. For every $0<s \leq$ $1-t_{0}, \lambda>0, a>0$, one has

$$
\mathbb{P}\left(T_{t_{0}}(a, s) \geq \lambda a s^{1-\frac{1}{\beta}}\right) \leq e^{-\lambda / 2 C}
$$

Proof Recall the notations $\mathbb{P}^{x}, \mathbb{E}^{x}$ in the proof of Lemma 7. By the Markov property,

$$
\mathbb{P}\left(T_{t_{0}}(a, s) \geq \lambda a s^{1-\frac{1}{\beta}} \mid \mathcal{F}_{t_{0}}\right)=g\left(M_{t_{0}}\right) \text { a.s. }
$$

where $g(x)=\mathbb{P}^{x}\left(T(a, s) \geq \lambda a s^{1-\frac{1}{\beta}}\right)$. It suffices to prove that the upper tail estimate

$$
\mathbb{P}^{x}\left(T(a, s) \geq \lambda a s^{1-\frac{1}{\beta}}\right) \leq e^{-\lambda / 2 C}
$$

holds uniformly for all $x \in \mathbb{R}^{1}$. To do so, let us compute the moment generating function of the sojourn time. First, we study its $n$-th moment for all $n \geq 2$. For every $k \in \mathbb{N}$ and $s \in \mathbb{R}^{+}$, let

$$
\Gamma_{k}=\Gamma_{k}(s)=\left\{\left(t_{1}, \ldots, t_{k}\right) \in[0, s]^{k}: 0 \leq t_{1} \leq \cdots \leq t_{k} \leq s\right\}
$$

Applying Fubini's theorem,

$$
\begin{aligned}
\mathbb{E}^{x} & {\left[T(a, s)^{n}\right] } \\
& =\int_{0}^{s} \cdots \int_{0}^{s} \mathbb{P}^{x}\left(\bigcap_{i=1}^{n}\left\{\left|M_{t_{i}}\right| \leq a\right\}\right) \mathrm{d} t_{1} \cdots \mathrm{d} t_{n}
\end{aligned}
$$




$$
\begin{aligned}
& =n ! \int_{\Gamma_{n}} \mathbb{P}^{x}\left(\bigcap_{i=1}^{n}\left\{\left|M_{t_{i}}\right| \leq a\right\}\right) \mathrm{d} t_{1} \cdots \mathrm{d} t_{n} \\
& \leq n ! \int_{\Gamma_{n}} \mathbb{P}^{x}\left(\bigcap_{i=1}^{n-1}\left\{\left|M_{t_{i}}\right| \leq a\right\},\left|M_{t_{n}}-M_{t_{n-1}}\right| \leq 2 a\right) \mathrm{d} t_{1} \cdots \mathrm{d} t_{n}
\end{aligned}
$$

which, by the Markov property, is equal to

$$
\begin{aligned}
& n ! \int_{\Gamma_{n}} \mathbb{E}^{x}\left[\mathbb{P}^{x}\left(\bigcap_{i=1}^{n-1}\left\{\left|M_{t_{i}}\right| \leq a\right\} \mid \mathcal{F}_{t_{n-1}}\right) \mathbb{P}^{M_{t_{n-1}}}\left(\left|M_{t_{n}}-M_{t_{n-1}}\right| \leq 2 a\right)\right] \\
& \quad \mathrm{d} t_{1} \cdots \mathrm{d} t_{n}
\end{aligned}
$$

Integrating over $t_{n}$, then pull out the conditional first moment of the sojourn time, one gets the upper bound

$$
\begin{aligned}
\mathbb{E}^{x}\left[T(a, s)^{n}\right] & \leq n ! \int_{\Gamma_{n-1}} \mathbb{E}^{x}\left[\mathbb{P}^{x}\left(\bigcap_{i=1}^{n-1}\left\{\left|M_{t_{i}}\right| \leq a\right\} \mid \mathcal{F}_{t_{n-1}}\right) \mathbb{E}^{M_{t_{n-1}}\left[T\left(2 a, s-t_{n-1}\right)\right]}\right] \\
& \mathrm{d} t_{1} \cdots \mathrm{d} t_{n-1} \\
\leq & n \cdot\left(\sup _{x \in \mathbb{R}^{d}} \mathbb{E}^{x}[T(2 a, s)]\right) \cdot(n-1) ! \int_{\Gamma_{n-1}} \mathbb{P}^{x}\left(\bigcap_{i=1}^{n-1}\left\{\left|M_{t_{i}}\right| \leq a\right\}\right) \mathrm{d} t_{1} \cdots \mathrm{d} t_{n-1} \\
= & n \cdot\left(\sup _{x \in \mathbb{R}^{d}} \mathbb{E}^{x}[T(2 a, s)]\right) \cdot \mathbb{E}^{x}\left[T(a, s)^{n-1}\right] .
\end{aligned}
$$

We iterate this procedure to get

$$
\mathbb{E}^{x}\left[T(a, s)^{n}\right] \leq n !\left(\sup _{x \in \mathbb{R}^{d}} \mathbb{E}^{x}[T(2 a, s)]\right)^{n}
$$

Thus for all $u>0$, the exponential moment of $T(a, s)$ is bounded from above by

$$
\mathbb{E}^{x}\left[e^{u T(a, s)}\right]=\sum_{n=0}^{+\infty} \frac{u^{n}}{n !} \mathbb{E}^{x}\left[T(a, s)^{n}\right] \leq \sum_{n=0}^{+\infty}\left(u \sup _{x \in \mathbb{R}^{d}} \mathbb{E}^{x}[T(2 a, s)]\right)^{n}
$$

Applying density estimate (6) yields for all $x \in \mathbb{R}^{d}, 0<s<1$,

$$
\mathbb{E}^{x}[T(2 a, s)]=\int_{0}^{s} \mathbb{P}^{x}\left(\left|M_{t}-x\right| \leq 2 a\right) \mathrm{d} t \leq \int_{0}^{s} t^{-1 / \underline{\beta}} \cdot 2 a \mathrm{~d} t \leq C \cdot a s^{1-\frac{1}{\beta}}
$$


with $C=\frac{2}{1-1 / \underline{\beta}}$, recalling that here $d=1$. Finally we choose

$$
u=\frac{1}{2 \sup _{x \in \mathbb{R}^{d}} \mathbb{E}^{x}[T(2 a, s)]}
$$

so that exponential moment (13) is bounded above by 1 . Consequently, using bound (14) and the Markov inequality yields that

$$
\mathbb{P}^{x}\left(T(a, s) \geq \lambda a s^{1-\frac{1}{\underline{\beta}}}\right) \leq e^{-u \lambda a s^{1-\frac{1}{\beta}}} \mathbb{E}^{x}\left[e^{u T(a, s)}\right] \leq e^{-\frac{\lambda}{2 C}}
$$

Since the estimate is uniform in $x$, the proof is complete.

The following density lemma is useful for our purpose. Comparing the usual mass distribution principle ([5, page 60]), it is the semi-time interval $[t, t+h]$ that is used rather than $[t-h, t+h]$ in order to apply the Markov property. We refer to [22, Lemma 4] for a proof. Recall that the Hausdorff measure of $E$ related to the gauge function $\varphi$ is defined by $\mathcal{H}^{\varphi}(E)=\lim _{\delta \downarrow 0} \mathcal{H}_{\delta}^{\varphi}(E)$ where

$$
\mathcal{H}_{\delta}^{\varphi}(E)=\inf \left\{\sum_{i} \varphi\left(\operatorname{diam}\left(Q_{i}\right)\right): E \subset \bigcup_{i} Q_{i} \text { with } \operatorname{diam}\left(Q_{i}\right) \leq \delta\right\}
$$

and $\varphi: \mathbb{R}_{+} \rightarrow \mathbb{R}_{+}$is an increasing function satisfying $\varphi(2 x) \leq K \varphi(x)$ around zero for some finite constant $K$.

Lemma 9 ([22]) Suppose that $v$ is a probability measure supported on $E \subset[0,1] \times \mathbb{R}$ such that for $v$-almost every $(t, x)$,

$$
\limsup _{h \rightarrow 0} \frac{v([t, t+h] \times[x-h, x+h])}{\varphi(h)} \leq C<+\infty .
$$

Then

$$
\mathcal{H}^{\varphi}(E) \geq \frac{1}{C}
$$

Proof of Proposition 3 Observe that the right-hand side term in (12) is 1 when $\beta \leq 1$. Using again the fact that the projection of a set in $\mathbb{R}^{2}$ to any line does not increase the Hausdorff dimension, we see that $\operatorname{dim}_{\mathcal{H}}\left(\operatorname{Gr}_{[0,1]}(M)\right) \geq \operatorname{dim}_{\mathcal{H}}([0,1])=1$, as desired.

Now consider $\beta>1$. For any $t_{0} \in[0,1)$, Lemma 8 applied to $a=s=2^{-m}$ and $\lambda=m$ so that $2^{-\bar{m}}<1-t_{0}$ yields that

$$
\mathbb{P}\left(T_{t_{0}}\left(2^{-m}, 2^{-m}\right) \geq m 2^{-m(2-1 / \underline{\beta})}\right) \leq e^{-m / 2 C} .
$$

We deduce using the Borel-Cantelli lemma that a.s. for all $m$ large enough,

$$
T_{t_{0}}\left(2^{-m}, 2^{-m}\right) \leq m 2^{-m(2-1 / \underline{\beta})} .
$$


For all $a$ small enough, let $m$ be the unique integer such that $2^{-m-1} \leq a<2^{-m}$. Then

$$
\frac{T_{t_{0}}(a, a)}{\log (1 / a) a^{2-1 / \underline{\beta}}} \leq \frac{T_{t_{0}}\left(2^{-m}, 2^{-m}\right)}{(\log 2) m 2^{-m(2-1 / \underline{\beta})}} \frac{2^{-m(2-1 / \underline{\beta})}}{2^{-(m+1)(2-1 / \underline{\beta})}} \leq C
$$

where $C$ is a positive finite constant independent of $m$. Thus for any $t_{0} \in[0,1)$, a.s.

$$
\limsup _{a \rightarrow 0} \frac{T_{t_{0}}(a, a)}{\log (1 / a) a^{2-1 / \underline{\beta}}} \leq C
$$

Consider the probability measure $\mu$, defined by $\mu(A):=\int_{0}^{1} 1_{A}\left(t, M_{t}\right) d t$ whose support is the graph $\operatorname{Gr}_{[0,1]}(M)$. Estimate (15) yields that for any fixed $t_{0} \in[0,1)$,

$$
\limsup _{a \rightarrow 0} \frac{\mu\left(\left[t_{0}, t_{0}+a\right] \times\left[M_{t_{0}}-a, M_{t_{0}}+a\right]\right)}{\log (1 / a) a^{2-1 / \underline{\beta}}} \leq C \quad \text { a.s. }
$$

A Fubini argument yields that a.s.

$$
\text { for Lebesgue a.e. } t \in(0,1), \quad \limsup _{a \rightarrow 0} \frac{\mu\left([t, t+a] \times\left[M_{t}-a, M_{t}+a\right]\right)}{\log (1 / a) a^{2-1 / \underline{\beta}}} \leq C
$$

Denote by $\mathcal{N} \subset[0,1]$ the Lebesgue null set such that (16) fails and set $G_{\mathcal{N}}=$ $\left\{\left(t, M_{t}\right) \in G r_{[0,1]}(M): t \in \mathcal{N}\right\}$, then

$$
\mu\left(G_{\mathcal{N}}\right)=\int_{0}^{1} 1_{G_{\mathcal{N}}}\left(t, M_{t}\right) \mathrm{d} t=0 .
$$

This, together with Lemma 9 applied to $\mu$, yields that a.s. $\mathcal{H}^{\varphi}\left(G r_{[0,1]}(M)\right) \geq 1 / C$ with $\varphi(x)=\log (1 / x) x^{2-1 / \underline{\beta}}$. The desired lower bound for the Hausdorff dimension of $\operatorname{Gr}_{[0,1]}(M)$ follows.

Finally we prove Theorem 2 when $d=1$.

Proof The upper bound is deduced from Proposition 2. Let us show the lower bound. To do so, we claim that for every $z \in \mathbb{R}$ and $t_{0} \in[0,1), \mathbb{P}^{z}$ a.s.,

$$
\operatorname{dim}_{\mathcal{H}} G r_{\left[t_{0}, 1\right]}(M) \geq \max \left(1,2-\frac{1}{\beta\left(M_{t_{0}}\right)}\right) .
$$

Consequently, $\mathbb{P}^{z}$ a.s. 


$$
\begin{aligned}
\operatorname{dim}_{\mathcal{H}}\left(G r_{[0,1]}(M)\right) & \geq \sup _{t_{0} \in[0,1] \cap \mathbb{Q}} \max \left(1,2-\frac{1}{\beta\left(M_{t_{0}}\right)}\right) \\
& =\max \left(1,2-\frac{1}{\sup _{t \in[0,1]} \beta\left(M_{t}\right)}\right)
\end{aligned}
$$

As the lower bound holds uniformly in $z \in \mathbb{R}$, the result follows.

It remains to prove the claim. Using the Markov property as in beginning of the proof of Lemma 7, it suffices to show that for any $x \in \mathbb{R}$,

$$
\mathbb{P}^{x}\left(\operatorname{dim}_{\mathcal{H}} G r_{\left[0,1-t_{0}\right]}(M) \geq 1 \vee(2-1 / \beta(x))\right)=1
$$

To this end, fix $x \in \mathbb{R}$ and consider the family of processes $\left\{M^{x, a} ; a<\beta(x)\right\}$ constructed in (10). Property (11) satisfied by $M^{x, a}$, together with Proposition 3 applied to the stable-like process with index function $\beta(\cdot) \vee a$, immediately implies (17) by letting $a \rightarrow \beta(x)$. The proof is now complete.

\section{Discussion}

This paper deals with the a typical family of Lévy-type processes with variable-order symbol. The SDE techniques used here allow to improve previously deterministic dimension bound to a stochastic one, and in the case of stable-like processes, the new bound is actually optimal. It would be interesting to see whether the SDE point of view allows to get dimension bounds for more general Lévy-type processes, in particular for those that do not have a density estimate like (6).

One possible extension of this article is the study of $\operatorname{dim}_{\mathcal{H}} M(E)$ with $E$ being any Borel set in $\mathbb{R}^{+}$. In [10], this question was considered and the authors obtained some bounds. The slicing and coupling argument of the present paper may certainly improve the bounds obtained in [10]. In one dimension, under monotonicity assumptions, quite precise answer to this question is given in [19].

Acknowledgements This work is a part of my PhD thesis at Université Paris-Est. I wish to thank my advisors Stéphane Jaffard and Stéphane Seuret for their constant support during the preparation of this paper. I also wish to thank Nicolas Fournier for stimulating discussions. I thank the anonymous referee for her/his careful reading and remarks which improved the quality of the manuscript.

\section{Appendix}

Here we close the gap in the proof of Proposition 1 . As the range of $\beta(\cdot)$ is included in a compact set of $(0,2)$, there exists $\varepsilon>0$ such that $x \mapsto \beta(x)$ is uniformly bounded from above by $2-\varepsilon$. Hence,

$$
\int_{\mathbb{S}^{d-1}} \int_{0}^{1}\left|\theta r^{1 / \beta(x)}\right|^{2} \frac{\mathrm{d} r}{r^{2}} H(\mathrm{~d} \theta)=\int_{0}^{1} r^{\frac{2}{2-\varepsilon}} \frac{\mathrm{d} r}{r^{2}}:=C<+\infty .
$$

The growth condition is thus satisfied. 
Let us now consider the Lipschitz condition. Let $x, y \in \mathbb{R}^{d}$. Without loss of generality, we assume $\beta(x)>\beta(y)$; then

$$
\begin{aligned}
& \int_{\mathbb{S}^{d-1}} \int_{0}^{1}\left|\theta r^{1 / \beta(x)}-\theta r^{1 / \beta(y)}\right|^{2} \frac{\mathrm{d} r}{r^{2}} H(\mathrm{~d} \theta) \\
& =\int_{0}^{1}\left(r^{1 / \beta(x)}-r^{1 / \beta(y)}\right)^{2} \frac{\mathrm{d} r}{r^{2}} \\
& =\int_{0}^{1} r^{2 / \beta(x)}\left(1-e^{\left(\log \frac{1}{r}\right)\left(\frac{1}{\beta(x)}-\frac{1}{\beta(y)}\right)}\right)^{2} \frac{\mathrm{d} r}{r^{2}} .
\end{aligned}
$$

Using the inequality $1-e^{-u} \leq u$ for $u>0$, this integral is bounded above by

$$
\begin{aligned}
& \int_{0}^{1} r^{2 / \beta(x)}\left(\log \frac{1}{r}\right)^{2}\left(\frac{1}{\beta(x)}-\frac{1}{\beta(y)}\right)^{2} \frac{\mathrm{d} r}{r^{2}} \\
& \leq C|x-y|^{2} \int_{0}^{1} r^{2 / \beta(x)}\left(\log \frac{1}{r}\right)^{2} \frac{\mathrm{d} r}{r^{2}},
\end{aligned}
$$

where we used the Lipschitz continuity of the function $\beta$. Remark that $\log (1 / r)^{2} \leq$ $C r^{-\varepsilon_{0}}$ for every $r \in(0,1)$ where $\varepsilon_{0}=\frac{1}{2}\left(\frac{2}{\sup _{x \in \mathbb{R}^{d}} \beta(x)}-1\right)$. Hence the last integral is finite and independent of $(x, y)$. The Lipschitz condition follows.

\section{References}

1. Bass, R.F.: Uniqueness in law for pure jump Markov processes. Probab. Theory Relat. Fields 79(2), 271-287 (1988)

2. Blumenthal, R.M., Getoor, R.K.: Some theorems on stable processes. Trans. Am. Math. Soc. 95, 263-273 (1960)

3. Blumenthal, R.M., Getoor, R.K.: The dimension of the set of zeros and the graph of a symmetric stable process. Ill. J. Math. 6, 308-316 (1962)

4. Böttcher, B., Schilling, R., Wang, J.: Lévy matters. III, volume 2099 of Lecture Notes in Mathematics. Springer, Cham, : Lévy-type processes: construction, approximation and sample path properties. With a short biography of Paul Lévy by Jean Jacod, Lévy Matters (2013)

5. Falconer, K.: Fractal Geometry. Mathematical Foundations and Applications, 2nd edn. Wiley, Hoboken (2003)

6. Fu, Z., Li, Z.: Stochastic equations of non-negative processes with jumps. Stoch. Process. Appl. 120(3), 306-330 (2010)

7. Jacob, N.: Pseudo Differential Operators and Markov Processes. Vol. III. Markov Processes and Applications. Imperial College Press, London (2005)

8. Jain, N., Pruitt, W.E.: The correct measure function for the graph of a transient stable process. Z. Wahrscheinlichkeitstheorie und Verw. Gebiete 9, 131-138 (1968)

9. Khoshnevisan, D., Xiao, Y., Zhong, Y.: Measuring the range of an additive Lévy process. Ann. Probab. 31(2), 1097-1141 (2003)

10. Knopova, V., Schilling, R.L., Wang, J.: Lower bounds of the Hausdorff dimension for the images of Feller processes. Statist. Probab. Lett. 97, 222-228 (2015)

11. Kolokoltsov, V.: Symmetric stable laws and stable-like jump-diffusions. Proc. Lond. Math. Soc. 80(3), 725-768 (2000)

12. Kolokoltsov, V.N.: Markov processes, semigroups and generators. de Gruyter Studies in Mathematics, vol. 38. Walter de Gruyter \& Co, Berlin (2011) 
13. Lépingle, D.: La variation d'ordre $p$ des semi-martingales. Z. Wahrscheinlichkeitstheorie und Verw. Gebiete 36(4), 295-316 (1976)

14. McKean Jr., H.P.: Sample functions of stable processes. Ann. Math. 61(2), 564-579 (1955)

15. Norvaiša, R., Salopek, D.M.: Estimating the $p$-variation index of a sample function: an application to financial data set. Methodol. Comput. Appl. Probab. 4(1), 27-53 (2002)

16. Pruitt, W.E., Taylor, S.J.: Sample path properties of processes with stable components. Z. Wahrscheinlichkeitstheorie und Verw. Gebiete 12, 267-289 (1969)

17. Pruitt, W.E.: The Hausdorff dimension of the range of a process with stationary independent increments. J. Math. Mech., 19:371-378, 1969/1970

18. Schilling, R.L.: Feller processes generated by pseudo-differential operators: on the Hausdorff dimension of their sample paths. J. Theoret. Probab. 11(2), 303-330 (1998)

19. Seuret, S., Yang, X.: Multifractal analysis for the occupation measure of stable-like processes. Electron. J. Probab. 22, 1-36 (2017)

20. Situ, R.: Theory of stochastic differential equations with jumps and applications. Mathematical and Analytical Techniques with Applications to Engineering. Springer, New York, 2005. Mathematical and analytical techniques with applications to engineering

21. Taylor, S.J.: The Hausdorff $\alpha$-dimensional measure of Brownian paths in $n$-space. Proc. Cambridge Philos. Soc. 49, 31-39 (1953)

22. Taylor, S.J., Wendel, J.G.: The exact Hausdorff measure of the zero set of a stable process. Z. Wahrscheinlichkeitstheorie und Verw. Gebiete 6, 170-180 (1966)

23. Tsuchiya, M.: Lévy measure with generalized polar decomposition and the associated SDE with jumps. Stoch. Stoch. Rep. 38(2), 95-117 (1992)

24. Xiao, Y.: Random fractals and Markov processes. In Fractal geometry and applications: a jubilee of Benô̂ t Mandelbrot, Part 2, vol. 72 of Proceedings of Symposia Pure Mathematics, pp. 261-338. American Mathematical Society, Providence, (2004)

25. Yang, X.: Multifractality of jump diffusion processes. Arxiv, e-print, (2015) 